Acta Crystallographica Section E

Structure Reports

Online

ISSN 1600-5368

\section{Diazidobis\{2-[3-(dimethylamino)propyl- iminomethyl]phenol\}manganese(III) perchlorate}

\section{Gui-Bin Yang* and Zhen-Hai Sun}

School of Chemistry and Life Sciences, Harbin University, Harbin 150080, People's Republic of China

Correspondence e-mail: ygb_hu@sina.com

Received 8 May 2008; accepted 24 July 2008

Key indicators: single-crystal X-ray study; $T=293 \mathrm{~K}$; mean $\sigma(\mathrm{C}-\mathrm{C})=0.005 \AA$; $R$ factor $=0.044 ; w R$ factor $=0.145 ;$ data-to-parameter ratio $=14.6$.

The title compound, $\left[\mathrm{Mn}\left(\mathrm{N}_{3}\right)_{2}\left(\mathrm{C}_{12} \mathrm{H}_{18} \mathrm{~N}_{2} \mathrm{O}\right)_{2}\right] \mathrm{ClO}_{4}$, was synthesized from manganese(III) acetate, sodium azide and 2-[3-(dimethylamino)propyliminomethyl]phenol by a hydrothermal reaction. The $\mathrm{Mn}^{\mathrm{III}}$ ion is hexacoordinated by two $\mathrm{N}$ and two $\mathrm{O}$ atoms from two phenolate ligands and two $\mathrm{N}$ atoms from two azide ligands. The $\mathrm{Mn}^{\mathrm{III}}$ cation lies on an inversion centre and, as a result, the asymmetric unit comprises one halfmolecule.

\section{Related literature}

For related literature, see: Choudhury et al. (2001); Church \& Halvorson (1959); Chung et al. (1971); Okabe \& Oya (2000); Serre et al. (2005); Scapin et al. (1997).

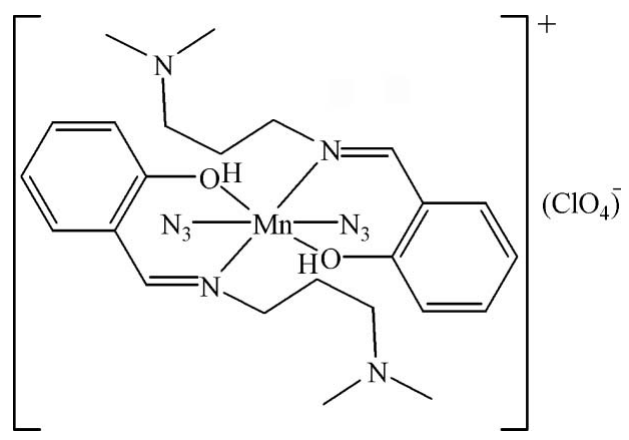

\section{Experimental}

Crystal data

$\left[\mathrm{Mn}\left(\mathrm{N}_{3}\right)_{2}\left(\mathrm{C}_{12} \mathrm{H}_{18} \mathrm{~N}_{2} \mathrm{O}\right)_{2}\right] \mathrm{ClO}_{4}$

$M_{r}=651.02$

Monoclinic, $C 2 / c$

$a=16.8115(17) \AA$

$b=16.4456(18) \AA$

$c=12.9059$ (14) $\mathrm{A}$

$\beta=121.121(8)^{\circ}$

$V=3054.6(6) \AA^{3}$

$Z=4$

Mo $K \alpha$ radiation

$\mu=0.57 \mathrm{~mm}^{-1}$

$T=293(2) \mathrm{K}$

$0.43 \times 0.28 \times 0.22 \mathrm{~mm}$

\section{Data collection}

Bruker APEXII CCD area-detector diffractometer

Absorption correction: multi-scan (SADABS; Bruker, 2001)

$T_{\min }=0.790, T_{\max }=0.884$

Refinement

$R\left[F^{2}>2 \sigma\left(F^{2}\right)\right]=0.043$

$w R\left(F^{2}\right)=0.144$

$S=1.00$

2842 reflections

195 parameters

3388 measured reflections 2842 independent reflections 2216 reflections with $I>2 \sigma(I)$ $R_{\text {int }}=0.044$

$\mathrm{H}$ atoms treated by a mixture of independent and constrained refinement $\Delta \rho_{\max }=0.48{\mathrm{e} \AA^{-3}}^{-3}$ $\Delta \rho_{\min }=-0.48{\mathrm{e} \AA^{-3}}^{-3}$

Data collection: APEX2 (Bruker, 2004); cell refinement: SAINTPlus (Bruker, 2001); data reduction: SAINT-Plus; program(s) used to solve structure: SHELXS97 (Sheldrick, 2008); program(s) used to refine structure: SHELXL97 (Sheldrick, 2008); molecular graphics: SHELXTL (Sheldrick, 2008); software used to prepare material for publication: SHELXTL.

The authors thank Harbin University for financial support.

Supplementary data and figures for this paper are available from the IUCr electronic archives (Reference: EZ2129).

\section{References}

Bruker (2001). SAINT-Plus and SADABS. Bruker AXS Inc., Madison, Wisconsin, USA.

Bruker (2004). APEX2. Bruker AXS Inc., Madison, Wisconsin, USA.

Choudhury, C. R., Dey, S. K., Mondal, N., Mitra, S., Mahalli, S. O. G. \& Malik, K. M. A. (2001). J. Chem. Crystallogr. 31, 57-62.

Chung, L., Rajan, K. S., Merdinger, E. \& Crecz, N. (1971). Biophys. J. 11, 469475.

Church, B. D. \& Halvorson, H. (1959). Nature (London), 183, 124-125.

Okabe, N. \& Oya, N. (2000). Acta Cryst. C56, 1416-1417.

Scapin, G., Reddy, S. G., Zheng, R. \& Blanchard, J. S. (1997). Biochemistry, 36, 15081-15088.

Serre, C., Marrot, J. \& Feréy, G. (2005). Inorg. Chem. 44, 654-658.

Sheldrick, G. M. (2008). Acta Cryst. A64, 112-122. 


\section{supporting information}

Acta Cryst. (2008). E64, m1080 [doi:10.1107/S1600536808023349]

\section{Diazidobis\{2-[3-(dimethylamino)propyliminomethyl]phenol\}manganese(III) perchlorate}

\section{Gui-Bin Yang and Zhen-Hai Sun}

\section{S1. Comment}

In recent years, Schiff base ligands have been widely used as polydentate ligands that can coordinate to transition or rare earth ions yielding complexes with interesting properties that are useful in materials science (Church \& Halvorson, 1959; Chung et al., 1971) and in biological systems (Okabe \& Oya, 2000; Serre et al., 2005; Scapin et al., 1997). Herein, we report the synthesis and X-ray crystal structure analysis of the title compound, (I).

The molecular structure of (I) is shown in Fig. 1. The $\mathrm{Mn}^{\mathrm{IIII}}$ cation lies on an inversion centre, as a consequence the asymmetric unit comprises half of the molecule. The $\mathrm{Mn}^{\mathrm{III}}$ ion is hexacoordinated by two $\mathrm{N}$ and two $\mathrm{O}$ atoms from two 2-[3-(dimethylamino)propyliminomethyl]phenolate ligands and two $\mathrm{N}$ atoms from two azide ligands.

\section{S2. Experimental}

The title compound was synthesized according to the following two steps:

(i) Synthesis of the ligand: 2-[3-(dimethylamino)propyliminomethyl]phenol was prepared by refluxing 3-dimethylamino-1-propylamine $(1.0 \mathrm{mmol})$ and salicylaldehyde $(1.0 \mathrm{mmol})$ in ethanol $(25 \mathrm{ml})$ for two hours and used without further purification, according to the literature method (see: Choudhury et al., 2001).

(ii) Synthesis of the complex: A solution of sodium azide $(0.5 \mathrm{mmol})$ and sodium perchlorate $(0.05 \mathrm{mmol})$ in $5 \mathrm{ml}$ water was added to the ethanol solution of the ligand $(1.0 \mathrm{mmol})$. Then manganese(III) acetate dihydrate $(0.5 \mathrm{mmol}) \mathrm{in} 3 \mathrm{ml}$ water was added to the above mixture. A yellow mixture was obtained by refluxing for $3 \mathrm{~h}$ and was left to stand undisturbed. Upon slow evaporation at room temperature, light yellow prismatic crystals suitable for X-ray diffraction appeared three days later and were separated by filtration.

\section{S3. Refinement}

The $\mathrm{H}$ atom on $\mathrm{O} 1$ was located from a difference density map and was refined with a distance restraint of $\mathrm{d}(\mathrm{O}-\mathrm{H})=$ 0.82 (2) $\AA$. All other $\mathrm{H}$ atoms were placed in calculated positions with $\mathrm{C}-\mathrm{H}=0.93 \AA$ and $\mathrm{N}-\mathrm{H}=0.86 \AA$ and refined as riding with $U_{\text {iso }}(\mathrm{H})=1.2 U_{\text {eq }}($ carrier $)$. 


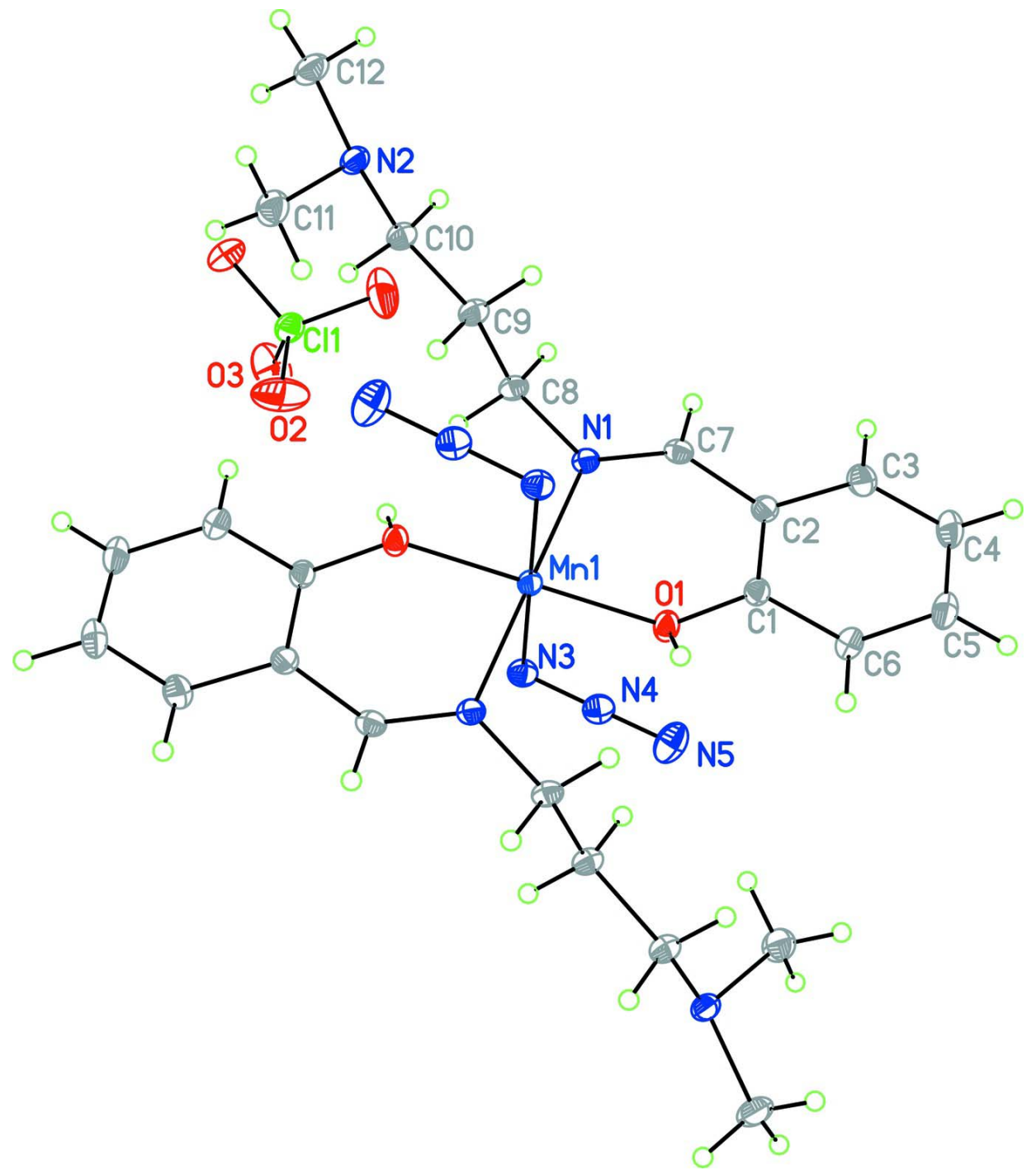

\section{Figure 1}

The molecular structure of (I), drawn with 30\% probability displacement ellipsoids for the non-hydrogen atoms.

Diazidobis\{2-[3-(dimethylamino)propyliminomethyl]phenol\}manganese(III) perchlorate

Crystal data

$\left[\mathrm{Mn}\left(\mathrm{N}_{3}\right)_{2}\left(\mathrm{C}_{12} \mathrm{H}_{18} \mathrm{~N}_{2} \mathrm{O}\right)_{2}\right] \mathrm{ClO}_{4}$

$M_{r}=651.02$

Monoclinic, $C 2 / c$

Hall symbol: $-\mathrm{C} 2 \mathrm{yc}$

$a=16.8115(17) \AA$

$b=16.4456(18) \AA$

$c=12.9059$ (14) $\AA$

$\beta=121.121(8)^{\circ}$

$V=3054.6(6) \AA^{3}$

$F(000)=1360$

$D_{\mathrm{x}}=1.416 \mathrm{Mg} \mathrm{m}^{-3}$

Mo $K \alpha$ radiation, $\lambda=0.71073 \AA$

Cell parameters from 2842 reflections

$\theta=1.9-25.5^{\circ}$

$\mu=0.58 \mathrm{~mm}^{-1}$

$T=293 \mathrm{~K}$

Prism, yellow

$Z=4$

$0.43 \times 0.28 \times 0.22 \mathrm{~mm}$ 


\section{Data collection}

Bruker APEXII CCD area-detector diffractometer

Radiation source: fine-focus sealed tube Graphite monochromator

$\varphi$ and $\omega$ scans

Absorption correction: multi-scan

(SADABS; Bruker, 2001)

$T_{\min }=0.790, T_{\max }=0.884$

\section{Refinement}

Refinement on $F^{2}$

Least-squares matrix: full

$R\left[F^{2}>2 \sigma\left(F^{2}\right)\right]=0.044$

$w R\left(F^{2}\right)=0.144$

$S=1.00$

2842 reflections

195 parameters

0 restraints

Primary atom site location: structure-invariant direct methods
3388 measured reflections

2842 independent reflections

2216 reflections with $I>2 \sigma(I)$

$R_{\text {int }}=0.044$

$\theta_{\text {max }}=25.5^{\circ}, \theta_{\min }=1.9^{\circ}$

$h=-20 \rightarrow 1$

$k=-1 \rightarrow 19$

$l=-13 \rightarrow 15$

Secondary atom site location: difference Fourier map

Hydrogen site location: inferred from neighbouring sites

$\mathrm{H}$ atoms treated by a mixture of independent and constrained refinement

$w=1 /\left[\sigma^{2}\left(F_{\mathrm{o}}^{2}\right)+(0.0843 P)^{2}+2.1116 P\right]$ where $P=\left(F_{\mathrm{o}}^{2}+2 F_{\mathrm{c}}^{2}\right) / 3$

$(\Delta / \sigma)_{\max }<0.001$

$\Delta \rho_{\max }=0.48$ e $\AA^{-3}$

$\Delta \rho_{\min }=-0.48$ e $\AA^{-3}$

\section{Special details}

Geometry. All e.s.d.'s (except the e.s.d. in the dihedral angle between two 1.s. planes) are estimated using the full covariance matrix. The cell e.s.d.'s are taken into account individually in the estimation of e.s.d.'s in distances, angles and torsion angles; correlations between e.s.d.'s in cell parameters are only used when they are defined by crystal symmetry. An approximate (isotropic) treatment of cell e.s.d.'s is used for estimating e.s.d.'s involving 1.s. planes.

Refinement. Refinement of $F^{2}$ against ALL reflections. The weighted $R$-factor $w R$ and goodness of fit $S$ are based on $F^{2}$, conventional $R$-factors $R$ are based on $F$, with $F$ set to zero for negative $F^{2}$. The threshold expression of $F^{2}>\sigma\left(F^{2}\right)$ is used only for calculating $R$-factors(gt) etc. and is not relevant to the choice of reflections for refinement. $R$-factors based on $F^{2}$ are statistically about twice as large as those based on $F$, and $R$ - factors based on ALL data will be even larger.

Fractional atomic coordinates and isotropic or equivalent isotropic displacement parameters $\left(\AA^{2}\right)$

\begin{tabular}{lllll}
\hline & $x$ & $y$ & $z$ & $U_{\text {iso }} * U_{\text {eq }}$ \\
\hline Mn1 & 0.2500 & 0.2500 & 0.5000 & $0.0467(2)$ \\
C11 & 0.0000 & $0.15323(8)$ & 0.7500 & $0.0711(3)$ \\
O1 & $0.28801(14)$ & $0.20397(12)$ & $0.40192(19)$ & $0.0626(5)$ \\
O2 & $0.0690(2)$ & $0.2006(3)$ & $0.7580(4)$ & $0.1579(17)$ \\
O3 & $0.0340(3)$ & $0.1044(2)$ & $0.8529(3)$ & $0.1482(15)$ \\
N1 & $0.13856(14)$ & $0.16698(13)$ & $0.43275(19)$ & $0.0481(5)$ \\
N2 & $-0.12645(16)$ & $0.32706(14)$ & $0.3901(2)$ & $0.0576(6)$ \\
N3 & $0.33339(17)$ & $0.16169(15)$ & $0.6490(2)$ & $0.0603(6)$ \\
N4 & $0.36786(18)$ & $0.10586(17)$ & $0.6266(2)$ & $0.0649(6)$ \\
N5 & $0.4004(3)$ & $0.0528(2)$ & $0.6034(4)$ & $0.0980(10)$ \\
C1 & $0.25785(18)$ & $0.14208(15)$ & $0.3267(2)$ & $0.0497(6)$ \\
C2 & $0.18274(18)$ & $0.09312(16)$ & $0.3069(2)$ & $0.0523(6)$ \\
C3 & $0.1561(2)$ & $0.0279(2)$ & $0.2252(3)$ & $0.0730(9)$ \\
H3A & 0.1071 & -0.0055 & 0.2123 & $0.088^{*}$ \\
C4 & $0.2015(3)$ & $0.0129(2)$ & $0.1639(4)$ & $0.0896(11)$
\end{tabular}




$\begin{array}{lllll}\text { H4A } & 0.1828 & -0.0300 & 0.1092 & 0.108^{*} \\ \text { C5 } & 0.2746(3) & 0.0614(2) & 0.1835(3) & 0.0791(10) \\ \text { H5A } & 0.3050 & 0.0512 & 0.1417 & 0.095^{*} \\ \text { C6 } & 0.3033(2) & 0.12473(19) & 0.2640(3) & 0.0633(7) \\ \text { H6A } & 0.3534 & 0.1565 & 0.2771 & 0.076^{*} \\ \text { C7 } & 0.12924(18) & 0.10820(16) & 0.3631(2) & 0.0513(6) \\ \text { H7A } & 0.0826 & 0.0708 & 0.3467 & 0.062^{*} \\ \text { C8 } & 0.06994(18) & 0.17367(16) & 0.4719(3) & 0.0532(6) \\ \text { H8A } & 0.1022 & 0.1806 & 0.5590 & 0.064^{*} \\ \text { H8B } & 0.0336 & 0.1241 & 0.4513 & 0.064^{*} \\ \text { C9 } & 0.0061(2) & 0.24542(17) & 0.4104(3) & 0.0572(7) \\ \text { H9A } & -0.0247 & 0.2389 & 0.3233 & 0.069^{*} \\ \text { H9B } & 0.0427 & 0.2949 & 0.4322 & 0.069^{*} \\ \text { C10 } & -0.0665(2) & 0.25332(17) & 0.4460(3) & 0.0583(7) \\ \text { H10A } & -0.1053 & 0.2051 & 0.4203 & 0.070^{*} \\ \text { H10B } & -0.0358 & 0.2570 & 0.5335 & 0.070^{*} \\ \text { C11 } & -0.0775(3) & 0.4036(2) & 0.4465(4) & 0.0850(10) \\ \text { H11A } & -0.0216 & 0.4066 & 0.4438 & 0.127^{*} \\ \text { H11B } & -0.0621 & 0.4052 & 0.5292 & 0.127^{*} \\ \text { H11C } & -0.1169 & 0.4488 & 0.4033 & 0.127^{*} \\ \text { C12 } & -0.2131(2) & 0.3213(2) & 0.3943(4) & 0.0844(11) \\ \text { H12A } & -0.2501 & 0.3691 & 0.3585 & 0.127^{*} \\ \text { H12B } & -0.1974 & 0.3170 & 0.4770 & 0.127^{*} \\ \text { H12C } & -0.2477 & 0.2742 & 0.3502 & 0.127^{*} \\ \text { H1A } & 0.313(2) & 0.2343(11) & 0.376(3) & 0.080^{*}\end{array}$

Atomic displacement parameters $\left(\AA^{2}\right)$

\begin{tabular}{lllllll}
\hline & $U^{11}$ & $U^{22}$ & $U^{33}$ & $U^{12}$ & $U^{13}$ & $U^{23}$ \\
\hline Mn1 & $0.0433(3)$ & $0.0501(3)$ & $0.0527(3)$ & $-0.0081(2)$ & $0.0290(3)$ & $-0.0109(2)$ \\
C11 & $0.0692(7)$ & $0.0852(8)$ & $0.0727(7)$ & 0.000 & $0.0465(6)$ & 0.000 \\
O1 & $0.0654(12)$ & $0.0654(12)$ & $0.0774(13)$ & $-0.0213(10)$ & $0.0513(11)$ & $-0.0260(10)$ \\
O2 & $0.083(2)$ & $0.220(4)$ & $0.168(4)$ & $-0.032(3)$ & $0.062(2)$ & $0.055(3)$ \\
O3 & $0.219(4)$ & $0.128(3)$ & $0.087(2)$ & $-0.018(3)$ & $0.071(2)$ & $0.0178(19)$ \\
N1 & $0.0422(11)$ & $0.0487(12)$ & $0.0532(12)$ & $-0.0003(9)$ & $0.0244(9)$ & $0.0002(10)$ \\
N2 & $0.0499(12)$ & $0.0601(14)$ & $0.0690(14)$ & $0.0035(11)$ & $0.0352(11)$ & $-0.0025(11)$ \\
N3 & $0.0555(13)$ & $0.0637(15)$ & $0.0616(14)$ & $-0.0029(12)$ & $0.0301(12)$ & $0.0023(12)$ \\
N4 & $0.0647(15)$ & $0.0638(16)$ & $0.0727(16)$ & $-0.0071(13)$ & $0.0401(14)$ & $0.0059(13)$ \\
N5 & $0.127(3)$ & $0.0709(19)$ & $0.135(3)$ & $0.0137(19)$ & $0.096(3)$ & $0.0101(19)$ \\
C1 & $0.0521(14)$ & $0.0471(13)$ & $0.0497(14)$ & $0.0050(11)$ & $0.0262(12)$ & $-0.0020(11)$ \\
C2 & $0.0484(14)$ & $0.0480(14)$ & $0.0532(14)$ & $0.0048(11)$ & $0.0212(12)$ & $-0.0027(11)$ \\
C3 & $0.073(2)$ & $0.0593(17)$ & $0.079(2)$ & $-0.0060(15)$ & $0.0346(17)$ & $-0.0179(16)$ \\
C4 & $0.102(3)$ & $0.079(2)$ & $0.094(3)$ & $-0.008(2)$ & $0.055(2)$ & $-0.039(2)$ \\
C5 & $0.095(3)$ & $0.080(2)$ & $0.079(2)$ & $0.006(2)$ & $0.057(2)$ & $-0.0181(18)$ \\
C6 & $0.0683(18)$ & $0.0650(17)$ & $0.0686(18)$ & $0.0052(15)$ & $0.0438(15)$ & $-0.0039(14)$ \\
C7 & $0.0425(13)$ & $0.0456(14)$ & $0.0567(15)$ & $-0.0028(11)$ & $0.0190(11)$ & $0.0005(12)$ \\
C8 & $0.0428(13)$ & $0.0569(15)$ & $0.0642(16)$ & $-0.0031(12)$ & $0.0307(12)$ & $0.0028(13)$ \\
C9 & $0.0483(15)$ & $0.0666(18)$ & $0.0628(17)$ & $0.0030(13)$ & $0.0329(13)$ & $0.0054(13)$
\end{tabular}




\begin{tabular}{lllllll}
$\mathrm{C} 10$ & $0.0521(16)$ & $0.0656(18)$ & $0.0652(17)$ & $0.0022(13)$ & $0.0359(14)$ & $0.0042(13)$ \\
$\mathrm{C} 11$ & $0.082(2)$ & $0.068(2)$ & $0.114(3)$ & $-0.0071(18)$ & $0.056(2)$ & $-0.019(2)$ \\
$\mathrm{C} 12$ & $0.0615(19)$ & $0.089(2)$ & $0.119(3)$ & $0.0021(18)$ & $0.059(2)$ & $-0.008(2)$ \\
\hline
\end{tabular}

Geometric parameters $\left(A,{ }^{\circ}\right)$

\begin{tabular}{|c|c|c|c|}
\hline $\mathrm{Mn} 1-\mathrm{O} 1$ & $1.8493(18)$ & $\mathrm{C} 3-\mathrm{C} 4$ & $1.377(5)$ \\
\hline $\mathrm{Mn} 1-\mathrm{O} 1^{\mathrm{i}}$ & $1.8493(18)$ & $\mathrm{C} 3-\mathrm{H} 3 \mathrm{~A}$ & 0.9300 \\
\hline $\mathrm{Mn} 1-\mathrm{N} 1^{\mathrm{i}}$ & $2.109(2)$ & $\mathrm{C} 4-\mathrm{C} 5$ & $1.374(5)$ \\
\hline $\mathrm{Mn} 1-\mathrm{N} 1$ & $2.109(2)$ & $\mathrm{C} 4-\mathrm{H} 4 \mathrm{~A}$ & 0.9300 \\
\hline $\mathrm{Mn} 1-\mathrm{N} 3^{\mathrm{i}}$ & $2.233(2)$ & $\mathrm{C} 5-\mathrm{C} 6$ & $1.370(4)$ \\
\hline $\mathrm{Mn} 1-\mathrm{N} 3$ & $2.233(2)$ & $\mathrm{C} 5-\mathrm{H} 5 \mathrm{~A}$ & 0.9300 \\
\hline $\mathrm{C} 11-\mathrm{O} 2$ & $1.357(3)$ & C6-H6A & 0.9300 \\
\hline $\mathrm{Cl} 1-\mathrm{O} 2^{\mathrm{ii}}$ & $1.357(3)$ & $\mathrm{C} 7-\mathrm{H} 7 \mathrm{~A}$ & 0.9300 \\
\hline $\mathrm{Cl} 1-\mathrm{O}^{3 i}$ & $1.397(3)$ & $\mathrm{C} 8-\mathrm{C} 9$ & $1.515(4)$ \\
\hline $\mathrm{C} 11-\mathrm{O} 3$ & $1.397(3)$ & $\mathrm{C} 8-\mathrm{H} 8 \mathrm{~A}$ & 0.9700 \\
\hline $\mathrm{O} 1-\mathrm{C} 1$ & $1.314(3)$ & $\mathrm{C} 8-\mathrm{H} 8 \mathrm{~B}$ & 0.9700 \\
\hline $\mathrm{O} 1-\mathrm{H} 1 \mathrm{~A}$ & $0.828(9)$ & $\mathrm{C} 9-\mathrm{C} 10$ & $1.516(4)$ \\
\hline $\mathrm{N} 1-\mathrm{C} 7$ & $1.273(3)$ & C9-H9A & 0.9700 \\
\hline $\mathrm{N} 1-\mathrm{C} 8$ & $1.483(3)$ & C9-H9B & 0.9700 \\
\hline $\mathrm{N} 2-\mathrm{C} 11$ & $1.474(4)$ & $\mathrm{C} 10-\mathrm{H} 10 \mathrm{~A}$ & 0.9700 \\
\hline $\mathrm{N} 2-\mathrm{C} 12$ & $1.489(4)$ & $\mathrm{C} 10-\mathrm{H} 10 \mathrm{~B}$ & 0.9700 \\
\hline $\mathrm{N} 2-\mathrm{C} 10$ & $1.502(4)$ & $\mathrm{C} 11-\mathrm{H} 11 \mathrm{~A}$ & 0.9600 \\
\hline $\mathrm{N} 3-\mathrm{N} 4$ & $1.199(4)$ & $\mathrm{C} 11-\mathrm{H} 11 \mathrm{~B}$ & 0.9600 \\
\hline $\mathrm{N} 4-\mathrm{N} 5$ & $1.149(4)$ & $\mathrm{C} 11-\mathrm{H} 11 \mathrm{C}$ & 0.9600 \\
\hline $\mathrm{C} 1-\mathrm{C} 6$ & $1.400(4)$ & $\mathrm{C} 12-\mathrm{H} 12 \mathrm{~A}$ & 0.9600 \\
\hline $\mathrm{C} 1-\mathrm{C} 2$ & $1.405(4)$ & $\mathrm{C} 12-\mathrm{H} 12 \mathrm{~B}$ & 0.9600 \\
\hline $\mathrm{C} 2-\mathrm{C} 3$ & $1.405(4)$ & $\mathrm{C} 12-\mathrm{H} 12 \mathrm{C}$ & 0.9600 \\
\hline $\mathrm{C} 2-\mathrm{C} 7$ & $1.439(4)$ & & \\
\hline $\mathrm{O} 1-\mathrm{Mn} 1-\mathrm{O} 1^{\mathrm{i}}$ & $180.00(8)$ & $\mathrm{C} 5-\mathrm{C} 4-\mathrm{H} 4 \mathrm{~A}$ & 120.0 \\
\hline $\mathrm{O} 1-\mathrm{Mn} 1-\mathrm{N} 1^{\mathrm{i}}$ & $89.94(8)$ & $\mathrm{C} 3-\mathrm{C} 4-\mathrm{H} 4 \mathrm{~A}$ & 120.0 \\
\hline $\mathrm{O} 1^{\mathrm{i}}-\mathrm{Mn} 1-\mathrm{N} 1^{\mathrm{i}}$ & $90.06(8)$ & $\mathrm{C} 6-\mathrm{C} 5-\mathrm{C} 4$ & $120.8(3)$ \\
\hline $\mathrm{O} 1-\mathrm{Mn} 1-\mathrm{N} 1$ & $90.06(8)$ & $\mathrm{C} 6-\mathrm{C} 5-\mathrm{H} 5 \mathrm{~A}$ & 119.6 \\
\hline 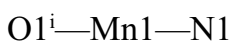 & $89.94(8)$ & $\mathrm{C} 4-\mathrm{C} 5-\mathrm{H} 5 \mathrm{~A}$ & 119.6 \\
\hline $\mathrm{N} 1{ }^{\mathrm{i}}-\mathrm{Mn} 1-\mathrm{N} 1$ & $180.00(13)$ & $\mathrm{C} 5-\mathrm{C} 6-\mathrm{C} 1$ & $120.7(3)$ \\
\hline $\mathrm{O} 1-\mathrm{Mn} 1-\mathrm{N} 3^{\mathrm{i}}$ & $87.82(10)$ & $\mathrm{C} 5-\mathrm{C} 6-\mathrm{H} 6 \mathrm{~A}$ & 119.7 \\
\hline $\mathrm{O} 1^{\mathrm{i}-}-\mathrm{Mn} 1-\mathrm{N} 3^{\mathrm{i}}$ & $92.18(10)$ & $\mathrm{C} 1-\mathrm{C} 6-\mathrm{H} 6 \mathrm{~A}$ & 119.7 \\
\hline $\mathrm{N} 1^{\mathrm{i}}-\mathrm{Mn} 1-\mathrm{N} 3^{\mathrm{i}}$ & $87.83(8)$ & $\mathrm{N} 1-\mathrm{C} 7-\mathrm{C} 2$ & $127.3(2)$ \\
\hline $\mathrm{N} 1-\mathrm{Mn} 1-\mathrm{N} 3^{\mathrm{i}}$ & $92.17(8)$ & $\mathrm{N} 1-\mathrm{C} 7-\mathrm{H} 7 \mathrm{~A}$ & 116.4 \\
\hline $\mathrm{O} 1-\mathrm{Mn} 1-\mathrm{N} 3$ & $92.18(10)$ & $\mathrm{C} 2-\mathrm{C} 7-\mathrm{H} 7 \mathrm{~A}$ & 116.4 \\
\hline $\mathrm{O} 1{ }^{\mathrm{i}-\mathrm{Mn} 1-\mathrm{N} 3}$ & $87.82(10)$ & $\mathrm{N} 1-\mathrm{C} 8-\mathrm{C} 9$ & $110.2(2)$ \\
\hline $\mathrm{N} 1{ }^{\mathrm{i}}-\mathrm{Mn} 1-\mathrm{N} 3$ & $92.17(8)$ & $\mathrm{N} 1-\mathrm{C} 8-\mathrm{H} 8 \mathrm{~A}$ & 109.6 \\
\hline $\mathrm{N} 1-\mathrm{Mn} 1-\mathrm{N} 3$ & $87.83(8)$ & $\mathrm{C} 9-\mathrm{C} 8-\mathrm{H} 8 \mathrm{~A}$ & 109.6 \\
\hline $\mathrm{N} 3{ }^{\mathrm{i}}-\mathrm{Mn} 1-\mathrm{N} 3$ & 180.0 & $\mathrm{~N} 1-\mathrm{C} 8-\mathrm{H} 8 \mathrm{~B}$ & 109.6 \\
\hline $\mathrm{O} 2-\mathrm{Cl1}-\mathrm{O} 2^{\mathrm{ii}}$ & $109.9(5)$ & $\mathrm{C} 9-\mathrm{C} 8-\mathrm{H} 8 \mathrm{~B}$ & 109.6 \\
\hline $\mathrm{O} 2-\mathrm{Cl} 1-\mathrm{O}^{\mathrm{ii}}$ & $108.4(3)$ & $\mathrm{H} 8 \mathrm{~A}-\mathrm{C} 8-\mathrm{H} 8 \mathrm{~B}$ & 108.1 \\
\hline $\mathrm{O} 2^{\mathrm{ii}-}-\mathrm{Cl} 1-\mathrm{O}^{\mathrm{ii}}$ & $110.1(2)$ & $\mathrm{C} 8-\mathrm{C} 9-\mathrm{C} 10$ & $111.8(2)$ \\
\hline
\end{tabular}




$\begin{array}{llll}\mathrm{O} 2-\mathrm{C} 11-\mathrm{O} 3 & 110.1(2) & \mathrm{C} 8-\mathrm{C} 9-\mathrm{H} 9 \mathrm{~A} & 109.2 \\ \mathrm{O} 2-\mathrm{C} 11-\mathrm{O} 3 & 108.4(3) & \mathrm{C} 10-\mathrm{C} 9-\mathrm{H} 9 \mathrm{~A} & 109.2 \\ \mathrm{O} 3-\mathrm{C} 11-\mathrm{O} 3 & 109.8(3) & \mathrm{C} 8-\mathrm{C} 9-\mathrm{H} 9 \mathrm{~B} & 109.3 \\ \mathrm{C} 1-\mathrm{O} 1-\mathrm{Mn} 1 & 133.21(18) & \mathrm{C} 10-\mathrm{C} 9-\mathrm{H} 9 \mathrm{~B} & 109.3 \\ \mathrm{C} 1-\mathrm{O} 1-\mathrm{H} 1 \mathrm{~A} & 104.7(14) & \mathrm{H} 9 \mathrm{~A}-\mathrm{C} 9-\mathrm{H} 9 \mathrm{~B} & 107.9 \\ \mathrm{Mn} 1-\mathrm{O} 1-\mathrm{H} 1 \mathrm{~A} & 117.3(13) & \mathrm{N} 2-\mathrm{C} 10-\mathrm{C} 9 & 111.7(2) \\ \mathrm{C} 7-\mathrm{N} 1-\mathrm{C} 8 & 117.6(2) & \mathrm{N} 2-\mathrm{C} 10-\mathrm{H} 10 \mathrm{~A} & 109.3 \\ \mathrm{C} 7-\mathrm{N} 1-\mathrm{Mn} 1 & 122.76(18) & \mathrm{C} 9-\mathrm{C} 10-\mathrm{H} 10 \mathrm{~A} & 109.3 \\ \mathrm{C} 8-\mathrm{N} 1-\mathrm{Mn} 1 & 119.59(17) & \mathrm{N} 2-\mathrm{C} 10-\mathrm{H} 10 \mathrm{~B} & 109.3 \\ \mathrm{C} 11-\mathrm{N} 2-\mathrm{C} 12 & 110.1(3) & \mathrm{C}-\mathrm{C} 10-\mathrm{H} 10 \mathrm{~B} & 109.3 \\ \mathrm{C} 11-\mathrm{N} 2-\mathrm{C} 10 & 112.8(2) & \mathrm{H} 10 \mathrm{~A}-\mathrm{C} 10-\mathrm{H} 10 \mathrm{~B} & 107.9 \\ \mathrm{C} 12-\mathrm{N} 2-\mathrm{C} 10 & 111.0(3) & \mathrm{N} 2-\mathrm{C} 11-\mathrm{H} 11 \mathrm{~A} & 109.5 \\ \mathrm{~N} 4-\mathrm{N} 3-\mathrm{Mn} 1 & 117.2(2) & \mathrm{N} 2-\mathrm{C} 11-\mathrm{H} 11 \mathrm{~B} & 109.5 \\ \mathrm{~N} 5-\mathrm{N} 4-\mathrm{N} 3 & 179.0(3) & \mathrm{H} 11 \mathrm{~A}-\mathrm{C} 11-\mathrm{H} 11 \mathrm{~B} & 109.5 \\ \mathrm{O} 1-\mathrm{C} 1-\mathrm{C} 6 & 117.9(3) & \mathrm{N} 2-\mathrm{C} 11-\mathrm{H} 11 \mathrm{C} & 109.5 \\ \mathrm{O} 1-\mathrm{C} 1-\mathrm{C} 2 & 123.1(2) & \mathrm{H} 11 \mathrm{~A}-\mathrm{C} 11-\mathrm{H} 11 \mathrm{C} & 109.5 \\ \mathrm{C} 6-\mathrm{C} 1-\mathrm{C} 2 & 119.0(3) & \mathrm{H} 11 \mathrm{~B}-\mathrm{C} 11-\mathrm{H} 11 \mathrm{C} & 109.5 \\ \mathrm{C} 1-\mathrm{C} 2-\mathrm{C} 3 & 118.8(3) & \mathrm{N} 2-\mathrm{C} 12-\mathrm{H} 12 \mathrm{~A} & 109.5 \\ \mathrm{C} 1-\mathrm{C} 2-\mathrm{C} 7 & 123.1(2) & \mathrm{N} 2-\mathrm{C} 12-\mathrm{H} 12 \mathrm{~B} & 109.5 \\ \mathrm{C} 3-\mathrm{C} 2-\mathrm{C} 7 & 118.0(3) & \mathrm{H} 12 \mathrm{~A}-\mathrm{C} 12-\mathrm{H} 12 \mathrm{~B} & 109.5 \\ \mathrm{C} 4-\mathrm{C} 3-\mathrm{C} 2 & 120.7(3) & \mathrm{N} 2-\mathrm{C} 12-\mathrm{H} 12 \mathrm{C} & 109.5 \\ \mathrm{C} 4-\mathrm{C} 3-\mathrm{H} 3 \mathrm{~A} & 119.6 & \mathrm{H} 12 \mathrm{~A}-\mathrm{C} 12-\mathrm{H} 12 \mathrm{C} & 109.5 \\ \mathrm{C} 2-\mathrm{C} 3-\mathrm{H} 3 \mathrm{~A} & 119.6 & \mathrm{H} 12 \mathrm{~B}-\mathrm{C} 12-\mathrm{H} 12 \mathrm{C} & 109.5 \\ \mathrm{C} 5-\mathrm{C} 4-\mathrm{C} 3 & 119.9(3) & & \\ & & & \end{array}$

Symmetry codes: (i) $-x+1 / 2,-y+1 / 2,-z+1$; (ii) $-x, y,-z+3 / 2$. 